\title{
mpMRI of the Prostate (MR-Prostatography): Updated Recommendations of the DRG and BDR on Patient Preparation and Scanning Protocol
}

\author{
mpMRT der Prostata (MR-Prostatografie): Aktualisierte \\ Empfehlungen der DRG und des BDR zur Vorbereitung \\ und Durchführung
}

\section{Autoren}

Tobias Franiel ${ }^{1}$, Patrick Asbach², Dirk Beyersdorff ${ }^{3}$, Dirk Blondin ${ }^{4}, 5$, Sascha Kaufmann ${ }^{6}$, Ullrich Gerd Mueller-Lisse ${ }^{7}$, Michael Quentin ${ }^{8}$, Stefan Rödel ${ }^{9}$, Matthias Röthke ${ }^{10}$, Heinz-Peter Schlemmer ${ }^{11}$, Lars Schimmöller ${ }^{12}$

Vorstand der Deutschen Röntgengesellschaft e. V. (DRG)

Gerald Antoch, Stefan O. Schönberg, Jörg Barkhausen, Frank Anton, Stefan Neumann, Günter Layer, Arnd Dörfler, Friederike Körber, Johannes Weßling, Michael Wucherer

Vorstand des Berufsverbandes der Deutschen Radiologen (BDR)

Detlef Wujciak, Bernd Hamm, Klaus Hamm, Andreas Bollkämper, Sönke Schmidt, Hermann Helmsberger,

Wolfram Schaeben, Julian Köpke, Stefan Neumann

\section{Affiliations}

1 Institut für diagnostische und interventionelle Radiologie, Universitätsklinikum Jena, Deutschland

2 Klinik für Radiologie, Charité Campus Benjamin Franklin, Charité-Universitätsmedizin Berlin, Deutschland

3 Klinik und Poliklinik für Diagnostische und Interventionelle Radiologie und Nuklearmedizin, Universitätsklinikum Hamburg-Eppendorf, Hamburg, Germany

4 Klinik für Radiologie, Gefäßradiologie und Nuklearmedizin, Städtische Kliniken Mönchengladbach GmbH ElisabethKrankenhaus Rheydt, Mönchengladbach, Germany

5 Klinik für Radiologie, Gefäßradiologie und Nuklearmedizin, Städtische Kliniken Mönchengladbach, Germany

6 Institut für Diagnostische und Interventionelle Radiologie, Siloah St. Trudpert Klinikum, Pforzheim, Deutschland

7 Klinik und Poliklinik für Radiologie, Klinikum der LudwigMaximilians-Universität München, Deutschland

8 Centrum für Diagnostik und Therapie $\mathrm{GmbH}$, Medizinisches Versorgungszentrum CDT Strahleninstitut GmbH, Köln, Germany

9 Radiologische Klinik, Städtisches Klinikum Dresden, Germany

10 Conradia Radiologie und Nuklearmedizin, Conradia Hamburg MVZ GmbH, Hamburg, Germany

11 Radiologie, Deutsches Krebsforschungszentrum, Heidelberg, Germany

12 Institut für Diagnostische und Interventionelle Radiologie, Universitätsklinikum Düsseldorf, Düsseldorf, Germany

Key words

prostate, MR-imaging, genital/reproductive, MR-diffusion/ perfusion, technical aspects received 16.02.2021

accepted 04.03.2021

published online 18.03.2021

Bibliography

Fortschr Röntgenstr 2021; 193: 763-776

DOI 10.1055/a-1406-8477

ISSN 1438-9029

(c) 2021. Thieme. All rights reserved.

Georg Thieme Verlag KG, Rüdigerstraße 14,

70469 Stuttgart, Germany

Correspondence

Prof. Dr. med. Tobias Franiel

Department of Diagnostic and Interventional Radiology,

University Hospital Jena, Am Klinikum 1, 07747 Jena,

Germany

Tel.: $+49 / 3641 / 9324831$

Fax: $+49 / 3641 / 9324832$

tobias.franiel@med.uni-jena.de

$\circledast$ Supplementary material is available under https://doi.org/10.1055/a-1406-8477

\section{ABSTRACT}

The Working Group Uroradiology and Urogenital Diagnosis of the German Roentgen Society (DRG) revised and updated the recommendations for preparation and scanning protocol of the multiparametric MRI of the Prostate in a consensus process and harmonized it with the managing board of German Roentgen Society and Professional Association of the German Radiologist (BDR e. V.). These detailed recommendation define the referenced "validated quality standards" of the 
German S3-Guideline Prostate Cancer and describe in detail the topic 1 . anamnestic datas, 2. termination of examinations and preparation of examinations, 3. examination protocol and 4. MRI-(in-bore)-biopsy.

Key Points:

- The recommendations for preparation and scanning protocol of the multiparametric MRI of the Prostate were revised and updated in a consensus process and harmonized with the managing board of GermanRoentgen Society (DRG) and Professional Asssociation of the German Radiologist (BDR).

- Detailed recommendations are given for topic 1. anamnestic datas, 2. termination and preparation of examinations, 3. examination protocoll and 4. MRI-(in-bore)biopsy.

- These recommendations define the referenced "validated quality standards" of the German S3-Guideline Prostate Cancer.

\section{Citation Format}

- Franiel T, Asbach P, Beyersdorff D et al. mpMRI of the Prostate (MR-Prostatography): Updated Recommendations of the DRG and BDR on Patient Preparation and Examination Protocol. Fortschr Röntgenstr 2021; 193: 763-776

\section{ZUSAMMENFASSUNG}

Die AG Uroradiologie und Urogenitaldiagnostik der Deutschen Röntgengesellschaft hat die Empfehlungen zur Vorbereitung und Durchführung der multiparametrischen MRT der Prostata im Konsensusverfahren und in Abstimmung mit den Vorständen der Deutschen Röntgengesellschaft und des Berufsverbandes der Deutschen Radiologen überarbeitet und aktualisiert. Diese aktualisierten Empfehlungen definieren die in der deutschen S3-Leitlinie Prostatakarzinom referenzierten "geltenden Qualitätsstandards“ und gehen detailliert auf die Themen 1. Anamnestische Angaben, 2. Untersuchungsterminierung und -vorbereitung, 3. Untersuchungsprotokoll und 4. MRT- (in-bore) -Biopsie ein.

\section{Introduction}

In recent years, multiparametric MRI (mpMRI) of the prostate (MR prostatography) has become firmly established in the outpatient and clinical routine of radiology. Technical and scientific progress in this field made it necessary to critically review the 2017 recommendations of the German Radiological Society (DRG) on the preparation and performance of MRI of the prostate and to adapt them accordingly. As part of this work, the recommendations of the DRG and the recommendations of the Professional Association of German Radiologists (BDR) for the sequence parameters of mpMRI of the prostate should be mutually harmonized at the same time. During 24 conference calls (60-90 min) between February 2020 and January 2021, members of both professional associations critically revised the 2017 recommendations (DRG: P.A., D.Be., D.Bl., T.F., S.K., U.M-L., M.Q., S.R., M.R., L.S., HP.S.; BDR: P. A., T.F., HP.S.). These updated recommendations relate to the preparation and performance of mpMRI of the prostate and MRI inbore biopsy and define the applicable quality standards of mpMRI of the prostate referenced in the German S3 Prostate Cancer Guideline ( $\triangleright$ Table 1 ). These recommendations have deliberately omitted guidance on reporting; in this regard, reference is made to the current PI-RADS guideline. However, it should be noted that although the recommendations share a large common intersection with the PI-RADS recommendations, they are not identical to them. Compared with the current PI-RADS guideline (v2.1), the explanations on case history information (Section 1) and on examination scheduling and preparation (Section 2) are more detailed. In addition, there are individual differences in the examination protocol (Section 3) regarding the recommended sequence parameters, which are meaningful from the authors' point of view, and the MRI in-bore biopsy (Section 4) is discussed in detail in contrast to the PI-RADS recommendations. During the composition of the authors team, care was taken to ensure that they are active members of the DRG Uroradiology and Genitourinary Diagnostics Working Group (WG) and have been scientifically involved in $\mathrm{mpMRI}$ of the prostate for many years. In addition, some authors are also members of the DRG Oncological Imaging Working Group (U.M-L., S.R., M.R., L.S., HP.S.). At the same time, care was taken to ensure that the authors were radiologists working in university hospitals (P.A., D.Be., T.F., S.K., U.M-L., L.S., HP.S.), in maximum and central care hospitals (D.BI., S.K., S.R.), and in private practice (P.A., M.Q., M.R.).

\section{Background Information}

\section{Case history data}

The risk of developing prostate cancer correlates positively with age, PSA level, PSA density, positive family history and palpation findings [1]. It has been shown that consideration of this patientspecific information in addition to the results of mpMRI of the prostate is associated with higher predictive accuracy for the presence of prostate cancer, lower number of negative biopsies, higher staging accuracy, and higher predictive accuracy for upgrading biopsy results [2-6].

\subsection{PSA}

Primary method for early detection is the determination of the total PSA level [1]. However, the PSA test is neither part of the statutory early detection procedure nor does it represent a benefit of the statutory health insurance funds for the early detection of prostate cancer. In practice, however, the PSA test is regularly offered as an "individual health service" (IGeL) in Germany. In the context of a screening examination, the determination of the PSA value thus represents an important building block for the decision-making process for or against biopsy of the prostate, 
- Table 1 Recommendations for the preparation and performance of mpMRI of the prostate (MR prostatography).

\section{Case history data}

\begin{tabular}{|c|c|}
\hline & Recommendations \\
\hline 1.1. PSA & $\begin{array}{l}\text { A current, and if possible, a confirmed total PSA value should be available. The progression of the PSA value should } \\
\text { also be available. PSA density should be calculated using the prostate volume determined on MRI. There should be } \\
\text { information on any medication affecting PSA levels. }\end{array}$ \\
\hline 1.2. Prior biopsies & $\begin{array}{l}\text { Information of prior prostate biopsies should be available, including type, extent, site of collection, and results of the } \\
\text { biopsies }\end{array}$ \\
\hline 1.3. Additional data & $\begin{array}{l}\text { The findings (if possible in combination with the corresponding DICOM image data) of previous mpMRIs of the } \\
\text { prostate should be available, as well as contact data of the referring physician. There should be information regarding } \\
\text { prior prostate-specific therapies. Risk factors for the presence of prostate cancer should be queried. }\end{array}$ \\
\hline $\begin{array}{l}\text { 1.4. Contrast agent risks } \\
\text { creatinine and eGFR }\end{array}$ & MRI contrast agents should be used in accordance with the current guidelines of the ESUR. \\
\hline 1.5. Implants & $\begin{array}{l}\text { There should be information regarding implants in the patient prior to the examination. The MRI examination should be } \\
\text { carried out in accordance with the applicable quality standards, taking into account the information in the implant ID. }\end{array}$ \\
\hline \multicolumn{2}{|c|}{ 2. Examination scheduling and preparation } \\
\hline & Recommendations \\
\hline 2.1. Time of examination & $\begin{array}{l}\text { An mpMRI of the prostate to find tumors before the first biopsy can be performed at any time. } \\
\text { An interval of at least } 6 \text { weeks between previous prostate biopsy and mpMRI of the prostate for tumor search/staging } \\
\text { should be aimed for. Patient-specific adaptation of the time frame can be made if clinically relevant. } \\
\text { Prostate mpMRI may be repeated at an interval of at least } 6 \text { weeks if the findings of mpMRI performed after prostate } \\
\text { biopsy are limited by hemorrhage, inflammation, or edema. }\end{array}$ \\
\hline 2.2. Antispasmotics & $\begin{array}{l}\text { To enhance image quality, } 20-40 \mathrm{mg} \text { of butylscopolamine should be applied IV slowly to reduce intestinal peristalsis } \\
\text { and fractionated if necessary. Alternatively or additionally, the administration can also be done intramuscularly. }\end{array}$ \\
\hline 2.3. Emptying the rectum & $\begin{array}{l}\text { The patient should be asked to empty the rectum and bladder prior to the examination. Enema or laxatives should not } \\
\text { be given directly before the examination, as these can increase intestinal peristalsis and exacerbate artifacts. }\end{array}$ \\
\hline 2.4. Abstinence & Insufficient evidence is available on the benefit of abstinence on detection/staging accuracy of MRI. \\
\hline \multicolumn{2}{|l|}{ 3. Examination protocol } \\
\hline & Recommendations/statements \\
\hline
\end{tabular}

For the issues of detection, staging and diagnosis of recurrence after radiation therapy and after prostatectomy, an identical, standardized, up-to-date protocol, both at 1.5 Tesla and 3 Tesla, should be used. This standard protocol consists of an axial T2w TSE sequence, a coronal and/or sagittal T2w TSE sequence, an axial DWI, an axial DCE sequence and a T1w sequence. Acquisition of the axial sequences (T2W, DWI and DCE sequences) should be made with the same slice thickness, the same angulation and the same number of slices if possible. Spatial resolution information for each sequence is given below. Here, the specified layer thicknesses and spatial resolutions in the in-plane level refer to measured and not to interpolated values.

Note: Additional sequences are to be completed according to the requirements of the Association of Statutory Health Insurance Physicians.

\begin{tabular}{l|l} 
3.1.T2W-TSE/FSE sequence & The T2w-TSE sequence should be acquired 2-dimensionally and at least bi-planar, whereby the axial plane is an
\end{tabular} obligatory component. A third plane increases the localization and staging accuracy.

The prostate should be centered and fully imaged in all planes. The bladder floor and the urogenital diaphragm should be fully imaged on at least 2 planes. The seminal vesicles and the lymph nodes in the obturator foramen should be completely imaged on at least one plane.

Axial:

Slice thickness $3.5 \mathrm{~mm}$ ( $3 \mathrm{~mm}$ preferred), $0 \%$ slice increment, FoV in phase $\leq 200 \mathrm{~mm}$, in-plane phase coding direction $\leq 0.8 \mathrm{~mm}$, in-plane frequency coding direction $\leq 0.6 \mathrm{~mm}$

Sagittal:

Slice thickness $3.5 \mathrm{~mm}$ ( $3 \mathrm{~mm}$ preferred), $0 \%$ slice increment, FoV in phase $\leq 200 \mathrm{~mm}$, in-plane phase coding direction $\leq 0.8 \mathrm{~mm}$, in-plane frequency coding direction $\leq 0.6 \mathrm{~mm}$

Coronal

Slice thickness $3.5 \mathrm{~mm}$ ( $3 \mathrm{~mm}$ preferred), $0 \%$ slice increment, FoV in phase $\leq 200 \mathrm{~mm}$, in-plane phase coding direction $\leq 0.8 \mathrm{~mm}$, in-plane frequency coding direction $\leq 0.6 \mathrm{~mm}$

3.2. DWI sequence To increase diagnostic accuracy, the DWI sequence should be an obligatory part of the protocol. The DWI sequence should be acquired axially: Slice thickness $3.5 \mathrm{~mm}$ ( $3 \mathrm{~mm}$ preferred), $0 \%$ slice increment, FoV in phase $\leq 200 \mathrm{~mm}$, in-plane phase coding direction $\leq 2.1 \mathrm{~mm}$, in-plane frequency coding direction $\leq 2.1 \mathrm{~mm}$ At least 2 different b-values should be measured to calculate the ADC map. b-value should lie between $0-100 \mathrm{~s} / \mathrm{mm}^{2}$ and another between $800-1000 \mathrm{~s} / \mathrm{mm}^{2}$ (preferably $1000 \mathrm{~s} / \mathrm{mm}^{2}$ ). In addition, a higher b-value $\geq 1400 \mathrm{~s} / \mathrm{mm}^{2}$ should also be measured or calculated. 
- Table 1 (Continuation)

3.3. DCE sequence According to current studies, a DCE sequence is of central importance for MRI diagnosis of prostate cancer.

The DCE sequence should be acquired axially:

Slice thickness $3.5 \mathrm{~mm}$ ( $3 \mathrm{~mm}$ preferred), $0 \%$ slice increment, FoV in phase $\leq 200 \mathrm{~mm}$, in-plane phase coding direction $\leq 2.1 \mathrm{~mm}$, in-plane frequency coding direction $\leq 2.1 \mathrm{~mm}$

The temporal resolution should be $\leq 9 \mathrm{~s}$ ( $\leq 6 \mathrm{~s}$ preferred). The flow rate of the contrast medium administration and the subsequent $\mathrm{NaCl}$ bolus ( $\mathrm{min} .30 \mathrm{ml}$ ) should be $\geq 2.5 \mathrm{ml} / \mathrm{s}$. The duration of the acquisition should not be less than $2 \mathrm{~min}$ (preferably $3 \mathrm{~min}$ ).

3.4. T1w sequence $\quad$ For the assessment of the bone and the lymph nodes as well as the prostate with regard to bleeding, for example, a T1w sequence should be acquired to map the entire pelvis from the aortic bifurcation to the pelvic floor.

- Slice thickness $\leq 5 \mathrm{~mm}(2 \mathrm{D}) / \leq 2 \mathrm{~mm}(3 \mathrm{D})$, slice increment $\leq 10 \%$, in-plane resolution $\leq 2.0 \times 2.0 \mathrm{~mm}$

The FoV has to be adapted to the patient as needed.

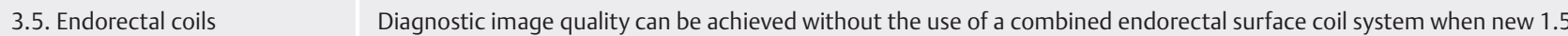
and 3 Tesla MR tomographs are employed.

If the image quality is not sufficient, a combined endorectal surface coil system can be used to increase the signal-tonoise ratio.

3.6. Additional sequences The sequences listed below are not intended to replace the routine protocol. They can also be used for the detection, localization and characterization of prostate cancer.

${ }^{1} \mathrm{H}-\mathrm{MRS}$

${ }^{1} \mathrm{H}-\mathrm{MRS}$ is an established method with a high level of evidence. Acquisition is via a $3 \mathrm{D}$ spin echo sequence. The peripheral zone should be completely covered by the ROI and the VOI should be significantly larger than the ROI.

The $3 \mathrm{D}$ acquisition matrix should include at least $8 \times 8 \times 8$ voxels (an interpolation of up to $16 \times 16 \times 16$ voxels should be aimed for).

The influence of tissues outside the prostate should be minimized by OVS. Signal contributions from water and lipids should be minimized.

As TR and TE have been particularly successful depending on the field strength: 1.5T: TR 1000 ms and TE 130ms; 3T: TR to $1000 \mathrm{~ms}$ and TE $145 \mathrm{~ms}$

\section{T2w 3 D multiecho sequences:}

The contrast properties of this sequence are not identical to those of a 2 D T2w TSE sequence. In addition, it shows increased susceptibility to motion artifacts. Because of the isotropic voxels, this sequence may be advantageous for fusion with other imaging modalities (e.g., ultrasound) and for contouring the prostate prior to planned radiotherapy. Diffusion tensor imaging, diffusion kurtosis imaging, BOLD imaging, MR elastography, T1 mapping, T2 mapping, ASL These techniques are the subject of research and should not be used outside of studies.

\section{MRI in-bore biopsy}

\section{Recommendations}

4.1. Indications and technique

A targeted MRI in-bore biopsy may be performed in individual cases for further clarification after negative systematic biopsy/fusion biopsy or as part of active surveillance/focal therapy. In the case of a primary indication/biopsy, deviations from the $\mathrm{S3}$ guideline should be clarified.

MRI in-bore biopsy should be performed transrectally as a standard procedure. Alternatively, it may be transgluteal or transperineal.

Transgluteal MRI in-bore biopsy is the method of choice in cases of rectal extirpation.

4.2. Laboratory procedures Prior to an MRT in-bore biopsy, a bleeding history should be recorded in a standardized manner. Current laboratory parameters should be determined according to the current recommendations of (CIRSE).

4.3. Anticoagulant medication The use of anticoagulant medication should be paused if possible. A dosage of $100 \mathrm{mg}$ ASA p. o. per day can be continued.

4.4. Antibiotics and anesthesia Transrectal MRI in-bore biopsy should be performed under antibiotic therapy.

MRI in-bore biopsy should be performed under local anesthesia (e. g. transrectally with gel containing lidocaine or transgluteal / transperineal with lidocaine injection s. c.).

ADC = apparent diffusion coefficient, $A S L=$ Arterial Spin Labeling, BOLD = Blood Oxygenation Level Dependent, CIRSE $=$ European Society for Cardiovascular and Interventional Radiology, DCE = Dynamic Contrast-Enhanced Imaging, DICOM = Digital Imaging and Communication in Medicine, DWI = Diffusionweighted Imaging, eGFR = estimated glomerular filtration rate, ESUR = European Society for Urogenital Radiology, FoV $=$ Field of View, Frequencyk. = Frequency coding direction, FSE = Fast Spin Echo, mpMRT = multiparametric magnetic resonance imaging, Phasenk. $=$ Phase coding direction, p. $\mathrm{o} .=$ per os, $\mathrm{PSA}=$ prostate specific antigen, $\mathrm{ROI}=$ region of interest, $\mathrm{s} . \mathrm{c}$. $=$ subcutaneous, $\mathrm{SD}=$ layer thickness, $\mathrm{T}=\mathrm{Tesla}, \mathrm{TE}=\mathrm{echo}$ time, $\mathrm{TR}=$ repetition time, $\mathrm{TSE}=$ turbo spin echo, $\mathrm{VOI}=$ volume of interest. 
whereas imaging procedures such as mpMRI of the prostate are not part of early detection screening. In contrast, mpMRI of the prostate has a high value in the primary diagnosis of suspiciously elevated PSA levels according to the recently updated S3 guideline for prostate cancer [1].

Therefore, if mpMRI of the prostate is planned, the PSA value should be available, if possible the confirmed PSA value (monitored for progression within one week using the same test procedure) [1]. Information on PSA level-influencing medications (e. g., 5 -alpha reductase inhibitors) or events (e.g., acute prostatitis) is also important. The most accurate determination of prostate volume is achieved with morphologic T2w sequences of mpMRI of the prostate. Calculation of PSA density [in $\mathrm{ng} / \mathrm{ml} / \mathrm{cm}^{3}$ ] should be performed using the MRI prostate volume and the associated PSA value.

\subsection{Prior biopsies}

Information (e.g., number, date, extent) of previously performed prostate biopsies should be available. Histopathologic results of previous biopsies (Gleason score or ISUP grading group) of a prostate cancer, the presence of prostatitis or benign prostatic hyperplasia (BPH), atypical microacinar proliferation (ASAP), or highgrade intraepithelial neoplasia of the prostate (HG-PIN) should be available and documented among the clinical information in the report of findings [1]. In addition, the biopsy cylinder collection site should be reported (including Gleason score and percent in the punch cylinder), as well as ASAP or HG PIN.

\subsection{Additional data}

\section{Prior examinations}

Information on previous examinations (palpation, transrectal ultrasound (TRUS), mpMRI of the prostate, and PSMA-PET-CT/MRI, etc.) should be requested and available at the time of evaluation of mpMRI of the prostate. Imaging studies should be available as DICOM data for comparison with current images. Contact information of the referring urologist/physician should be available for direct communication of results.

\section{Risk factors}

Risk factors with high evidence for the presence of prostate cancer are age and a positive family history [1]. The incidence of prostate cancer increases with age [7]; likewise, the incidence of prostate cancer correlates positively with a positive family history. The relative risk is increased by $2.5-4.3 \%$ for any first-degree relative $[1,8]$. Other factors that increase the relative risk of prostate cancer include: younger age of affected family members, increasing genetic match to the affected family member, and increasing number of individually affected family members [1]. Other possible risk factors (e. g., mutation in the BRCA1 or BRCA2 gene) are also under discussion, but their association with prostate cancer has not yet been sufficiently demonstrated.

\section{Anti-hormonal therapy}

The prostate is an androgen-sensitive organ. Anti-hormonal therapy results in decreased activity of glandular function associated with a reduction in prostate volume and signal reduction on the T2w image. Demarcation of the prostatic zones may be more difficult or even impossible. The effects of anti-hormonal therapy complicate tumor detection on T2w and DWI images. Under anti-hormonal therapy, the tumors are typically much smaller or no longer distinct. The effects of anti-hormonal therapy on the prostate as well as on prostate cancer are typically noticeable after a short period of treatment [9].

\section{Therapy with 5-alpha reductase inhibitors}

The volume of the peripheral and transition zones are reduced during treatment with 5-alpha reductase inhibitors [10].

\section{Radiotherapy}

After radiotherapy, zonal patterning is usually absent and the peripheral zone often exhibits extensive low T2w signal intensity. Volume reduction often occurs in the course of therapy. The contour and neurovascular bundle may be accentuated and the bladder and rectal walls thickened [11].

\section{Clinical prostatitis and information on its treatment}

Prostatitis can lead to extensive signal reductions on T2-weighted images, which can negate the zonal structure. Depending on the extent and type of inflammation, the differentiation of a coincident carcinoma may be difficult or impossible due to the marked changes. Prostatitis often shows extensive changes with sometimes asymmetric volume increase. Mild inflammation may be recognized based on the band, wing, wedge, or diffuse appearance, which is usually not focal. Abscesses (micro or macro) may be present in cases of bacterial inflammation. Prostatitis usually shows little diffusion restriction and early enhancement in a DCE sequence [12].

\section{Previous pelvic surgeries}

Transurethral resection (TUR-P) of BPH leaves a subtotal to total defect in the transition zone. The remaining peripheral zone typically shows extensive $\mathrm{T} 2 \mathrm{w}$-weighted signal intensity reductions, possibly with emphasis around the resection area. Since hyperplasia nodes occasionally develop again in the resection area, the time at which the TUR-P is performed and information on the success of the treatment, if available, are helpful. Information on focal therapy for prostate cancer should also be available. This includes the technique used, the timing and extent of treatment. After focal therapy, there is initial swelling and, in the further course, shrinkage of the treated area due to fibrosis and, depending on the focal therapy method used, possible cyst formation. Rectal resection and rectal amputation, as well as extensive repeated local treatment of bladder tumors, can alter the prostate and the tissue surrounding the prostate.

\subsection{Contrast agent risks, creatinine and eGFR}

Determination of renal function (creatinine and eGFR) may be performed prior to MRI contrast administration, but is not mandatory. The current guidelines of the European Society of Urogenital Radiology (ESUR) should be followed accordingly. If serum creati- 
nine has not been determined, renal function should be assessed by questionnaire.

Gadolinium-based contrast agents have a very low risk of causing acute or late reactions in the form of nephrotoxicity or other serious adverse effects after intravenous administration. However, it is known that small amounts of Gd-based contrast can be deposited in the body after use. The Committee for Medicinal Products for Human Use (CHMP) at the European Medicines Agency (EMA) has assessed the risk for Gd-based contrast and the occurrence of nephrogenic systemic fibrosis (NSF) as a basis for recommendations for use [13]. To date, there is no evidence of harm to patients from deposition of these contrast agents in the brain [14]. However, because the long-term risks are unknown, the EMA additionally recommended the suspension of marketing authorizations for intravenous linear Gd-based contrast agents in the EU [14]. Accordingly, for DCE sequences, only Gd-based contrast agents with cyclic chelate ligands (gadobutrol, gadoterate meglumine, gadoteridol) and thus lowest NSF risk are still allowed. High NSF risk is present in stage 4 and 5 chronic kidney dysfunction (CKD) (GFR $\left.<30 \mathrm{ml} / \mathrm{min} / 1.73 \mathrm{~m}^{2}\right)$, dialysis patients, or acute renal failure; low NSF risk is present in stage 3 CKD (GFR $30-59 \mathrm{ml} / \mathrm{min} / 1.73 \mathrm{~m}^{2}$ ); and no NSF risk is present in stable GFR $\geq 60 \mathrm{ml} / \mathrm{min} / 1.73 \mathrm{~m}^{2}$. In patients with stage 4 and 5 chronic kidney dysfunction (CKD), there should be at least seven days between injections [13].

According to the SmPC, the lowest dose of a Gd-based contrast, with assurance of adequate contrast enhancement, should always be used. The name and dosage of the contrast agent used should always be noted on a patient-specific basis. Acute adverse events can be identified and treated by monitoring the patient for a period of 30 minutes after contrast administration as well as having emergency medications and equipment available at the examination site [13].

In patients at increased risk for an adverse effect, contrast administration should be avoided or alternative procedures should be considered. If there is a history of known allergy to a specific Gd-based contrast agent, an alternative agent should be used first and premedication should be considered to prevent contrast intolerance. With hemodialysis patients, it is recommended that hemodialysis be performed as soon as possible after contrast administration.

\subsection{Implants}

Due to the different magnetic fields, body implants can pose different dangers and injury mechanisms for the patient in the MRI [15]. The B0 magnetic field can cause translational and rotational movements of the implants. The magnetic fields of the gradient coils can induce a current in the implants and the magnetic fields of the high-frequency coils cause the temperature of the implants to rise. This increase in temperature depends on the energy of the high-frequency pulses, the position of the implant in the high-frequency field and the ratio of the implant length to the wavelength of the radiated high-frequency pulse. For these reasons, patients with implants can only be safely examined in the MRI if the implants are identified as MR-safe or MR conditionally safe according to the implant ID and the manufacturer's specifications are com- plied with. For an economical execution of MRI workflows, it is therefore important to clarify the MRI safety of an implant in good time before the actual MRI examination.

In addition to the dangers described for the patient, implants lead to field inhomogeneities which, due to the associated image artifacts, make diagnostics difficult or even impossible. In this context, hip prostheses are a common problem in mpMRI of the prostate. Since the field inhomogeneities caused by implants escalate with increasing field strength, an examination of conditionally MRI-safe hip joint prostheses at $1.5 \mathrm{~T}$ may be useful in individual cases, depending on the respective $3 T$ MRI device and the examination/sequence quality. However, artifacts can occur at both field strengths, and the extent of artifacts generally cannot be predicted with confidence in practice.

The DWI sequence, which is important for diagnostics, is particularly susceptible to field inhomogeneities. Therefore, new acquisition techniques have been developed that result in less image distortion. These include DWI sequences with parallel imaging and selective two-dimensional excitation of a small examination volume (parallel transmitted EPI sequences) or multi-shot sequences with segmented readout and susceptibility artifact/ motion correction (readout segmented multishot EPI sequences) $[16,17]$ (refer to discussions in the DWI sequence section). Furthermore, changing the phase encoding direction, increasing the bandwidth, enlarging the matrix, reducing the slice thickness, reducing the TE time or using special artifact reduction sequences such as MAVRIC (multi-acquisition variable-resonance image combination) and SEMAC (slice encoding for metal artifact correction) can be helpful for artifact reduction [18].

\section{Examination scheduling and preparation}

\subsection{Time of examination}

Prior to an initial biopsy of the prostate, mpMRI can be performed to screen for tumors at any time. After a prostate biopsy has already been performed, intraprostatic bleeding, inflammation and edema can usually be found in the peripheral zone and in the seminal vesicles (depending on the number of biopsy cylinders removed, among other things). These changes may interfere with the assessment of mpMRI of the prostate in general and, in particular, with the assessment of the prostate pseudocapsule (glandfree layers of connective tissue and muscle cells), the neurovascular bundle, and the seminal vesicles. Therefore, MRI may be more appropriate at a later time when the changes have regressed or are undetectable. Although these changes may be detectable several months after biopsy, there is a reduction over time, so an interval of at least 6 weeks between previous prostate biopsy and prostate mpMRI should be aimed for. On the other hand, deferral is not always appropriate or necessary due to individual circumstances or clinical relevance. In this context, it should be taken into account that the probability of the presence of clinically significant prostate cancer in regions with hemorrhage without corresponding suspicious MRI changes after previous negative biopsy of the prostate is low $[19,20]$. 


\subsection{Antispasmotics}

The use of an intestinal spasmolytic leads to a relevant reduction of artifacts caused by the natural movement of the bowel and thus to an increase in image quality and should therefore be applied routinely [20-23]. Individual studies, but without a prospective comparison within a patient and in some cases with the use of an endorectal coil, assessed the effect to be lower [24, 25].

In the absence of contraindications, one to two ampoules of butylscopolamine (e. g., butylscopolaminium bromide, Busco$\left.\operatorname{pan}^{\circledR}\right)$ (20-40 mg) should be applied according to weight. Intravenous administration of butylscopolamine is followed by rapid distribution in the body with a plasma/distribution half-life of 4 min. The tissue/elimination half-life is 29 min. Thus, IV administration of the spasmolytic should occur approximately 4 min before the high-resolution axial T2w and DWI sequences to achieve maximal effect for these sequences. Additional IM administration or fractionated IV administration may be considered to prolong maximum efficacy. The terminal half-life of butylscopolamine is approximately 5.1 hours, so operating a motor vehicle should be avoided for 6 hours. Contraindications such as narrow-angle glaucoma and cardiac arrhythmias (e. g., tachycardia, tachyarrhythmia) must be ruled out. Further contraindications to the administration of butylscopolamine can be found in the Summary of Product Characteristics. BPH should be considered a relative contraindication. The bladder should be emptied prior to the examination. Acute urinary retention after administration of butylscopolamine is extremely rare with an empty bladder due to the short half-life and should be treated urologically in individual cases.

As an alternative to the administration of butylscopolamine, intravenous administration of one ampoule of glucagon $(1 \mathrm{mg})$ is possible, subject to contraindications. In addition, prior food abstinence (no food or fluids for 3-4 hours) may be considered. Currently, however, there is no evidence in the literature to support the use of glucagon or prior dietary restriction.

\subsection{Emptying the rectum}

A rectal ampulla filled with feces causes local field inhomogeneities due to rectal air at the interface with the peripheral zone of the prostate, which lead to image distortions that negatively affect the image quality of the DWI sequence in particular [26]. The feces-filled rectal ampulla further leads to increased rectal contractions, which in turn result in stronger movement artifacts [26]. The causally simplest measure to reduce and, in the best case, avoid these artifacts is to empty the rectum. Alternative but more complex measures include examination in the prone position and active removal of rectal air with a narrow catheter. Administration of a microenema directly before the examination, however, does not lead to an improved image quality or a reduction of image artifacts [27]. Technical options for artifact reduction include manual adjustment and reduction of the shim volume and the use of new acquisition techniques (see discussions in the section on implants and DWI sequences).

\subsection{Abstinence}

The term abstinence is understood to mean abstaining from ejaculations for a period of time prior to performance of an MRI. Studies have examined the behavior of T2w imaging in healthy subjects and found a T2w signal decrease in the peripheral zone after ejaculation [28, 29]. Another study in healthy subjects concluded that the ADC value in diffusion-weighted imaging post ejaculation is also reduced [30]. This effect was evident for over 24 hours; parameters normalized afterward. The authors concluded that abstinence for 3 days is recommended before mpMRI of the prostate to improve assessment of the seminal vesicles and increase staging accuracy [30]. A decrease in the volume of the seminal vesicles after ejaculation has been reported, but the temporal relationship between ejaculation and the volume of the seminal vesicles is described as being only slightly correlated [29, 31, 32].

In summary, the positive influence of abstinence on the detection rate of prostate cancer cannot be sufficiently proven, but abstinence on the evening before and on the day of the MRI seems to make sense. A binding recommendation for an abstinence interval cannot be given due to the current lack of sufficient studies.

\section{Examination protocol}

The layer thicknesses and spatial resolutions given in $>$ Table 1 and in this section always relate to measured and not interpolated values.

\subsection{T2w-TSE/FSE sequence}

T2w images with spatially high-resolution T2w turbo spin echo (TSE) or fast spin echo (FSE) sequences form the morphological basis of mpMRI of the prostate. The recommended layer thickness is $3.5 \mathrm{~mm}$ (preferably $3 \mathrm{~mm}$ ) and the slice increment is $0 \%$. When using a slice thickness of $3 \mathrm{~mm}$, a slice increment of $10 \%$ can also be selected, taking into account the slice profile sloping towards the edges and to increase the signal-to-noise ratio (SNR). The fat signal should not be suppressed. On this basis, a targeted biopsy can be performed in the next step. T2-weighted images of the prostate should always be made in the axial plane and supplemented by orthogonal T2-weighted images in at least one other plane [20]. Acquisition of the axial sequences (T2w, DWI and DCE sequences) should be made with the same slice thickness, the same angulation and the same number of slices if possible. During the conference, the authors worked out three different options for axial angulation:

1. Axially to urethra (preferred by 3 authors),

2. Axially to the longitudinal axis of the body (preferred by 2 authors) and

3. Axially to anterior rectal wall (preferred by 5 authors) ( $\triangleright$ Fig. 1).

Each of these angulations has advantages and disadvantages, which are listed in $>$ Table 2 . In this context, it is important that the selected angulation should be documented in the findings in order to avoid errors in the correlation with TRUS examinations, follow-up checks, image fusions and biopsy planning. The acquisition should be carried out with right-to-left phase coding direc- 
tion from to avoid potential movement artifacts of the rectum at the level of the prostate. The sagittal T2w sequence is acquired strictly sagittally to the longitudinal axis of the body (phase coding direction preferably head-toe to reduce breathing artifacts) and thus runs perpendicular to angulation 2 axially to the longitudinal axis of the body. Angulation of the coronal T2w sequence (phase encoding direction right-left) is parallel to the largest contact area of the peripheral zone with the rectal anterior wall and is perpendicular to angulation 3 axially to the rectal anterior wall ( $\triangleright$ Fig. 2). With this angulation, the alignment along the not always clearly identifiable ejaculatory duct is most likely achieved for the detection of seminal vesicle infiltration.

If the main focus for mpMRI of the prostate is the staging of prostate cancer, the prostate should preferably be examined in three planes with $\mathrm{T} 2 \mathrm{w}$ images and at least a higher axial resolution, namely in-plane in phase encoding direction $\leq 0.7 \mathrm{~mm} \times$ inplane in frequency encoding direction $\leq 0.5 \mathrm{~mm}$.

Further information on the anatomy and its implications for angulation of the examination planes and reporting can be found in the "Supplementary Material" section.

\subsection{DWI sequence}

Due to their increased cell density, prostate cancers shrink the interstitial space and displace, compress, or destroy the glands or glandular excretory ducts. This restricts the free mobility of particles (diffusion), which is represented graphically with the DWI sequence [33].

Single-shot echo planar imaging (SS-EPI) sequences are most commonly used for DWI sequencing. Image data sets are acquired with low diffusion weighting (low b-value between 0 and $100 \mathrm{~s} / \mathrm{mm}^{2}$ ) as well as with strong diffusion weighting (high b-value between 800 and $1000 \mathrm{~s} / \mathrm{mm}^{2}$ ). In clinical routine, the monoexponential model for calculating the so-called apparent diffusion coefficient (ADC) has become widely accepted. A monoexponential function is used to determine the ADC for each image point using the two image data sets with low and high b-values, respectively, and the numerical value is mapped as image contrast in ADC images [33]. For each pixel, a straight line is drawn through the two measured b-values. The ADC value is a pictorial representation of the slope of this straight line. The higher the SNR of the respective measured b-value images, the better the determination of the straight line. The SNR of b-value images can be positively influenced by increasing the number of averages and should be carefully optimized for each b-value depending on the MRI equipment (including receiving coil) available in each case. It is therefore particularly advisable to select the highest possible number of averages for the high measured b-value and to exclude the very high $b$-value $\left(>1400 \mathrm{~s} / \mathrm{mm}^{2}\right.$ ) from the calculation of the $A D C$ in order to avoid kurtosis effects influencing the ADC. If this high $b$-value is measured (and not calculated), it should therefore be recorded separately. A value above $0 \mathrm{~s} / \mathrm{mm}^{2}$ (e.g. 50$100 \mathrm{~s} / \mathrm{mm}^{2}$ ) can be preferred as the lowest b-value in order to reduce perfusion effects and their influence on the calculation of the ADC maps. A right-left phase coding direction can reduce breathing artifacts and optimize the image quality. However, the patient's hands/arms should not be on the side of the pelvis to

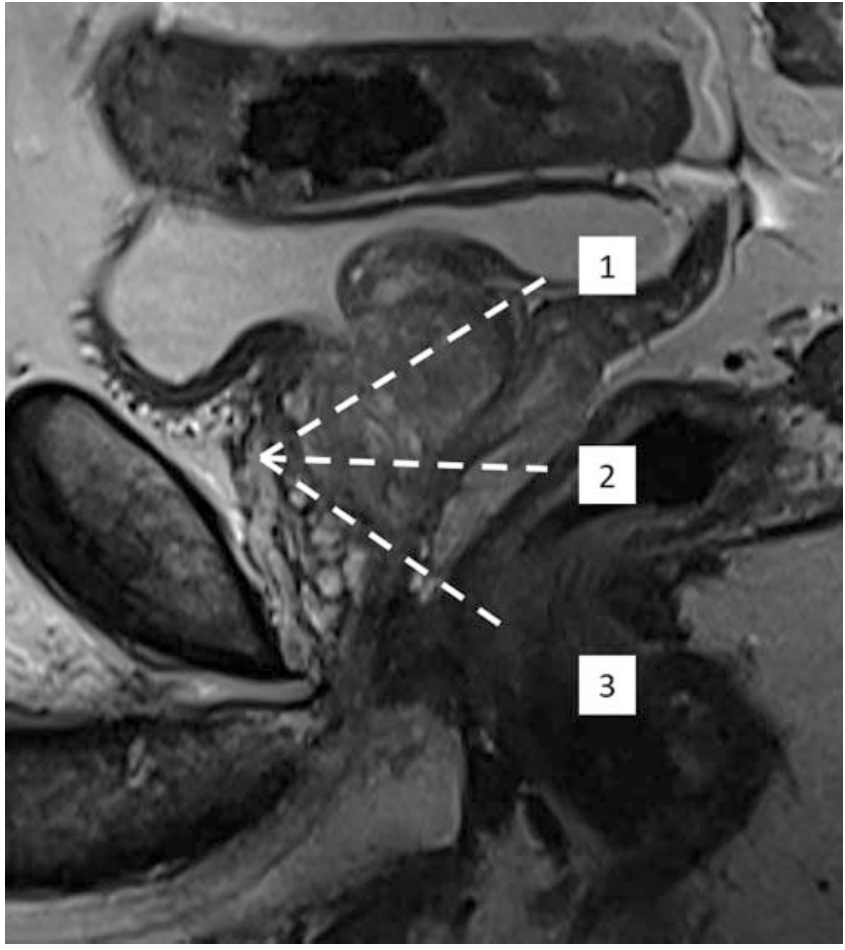

- Fig. 1 Sagittal T2-weighted image of the prostate for illustration of three possible axial angulations: 1 . axial to urethra, 2 . axial to longituidinal body axis and 3. axial to anterior rectal wall.

avoid folding (if phase oversampling is not used). An anterior-posterior phase coding direction can, in turn, possibly enable better diagnostic images in the case of hip implants.

Several new sequencing techniques can reduce the susceptibility of common fat-saturated SS-EPI sequences to artifacts, distortions due to magnetic field inhomogeneity during susceptibility jumps, e. g., at tissue boundaries to air or metallic implants. These include sequences with parallel imaging and selective two-dimensional excitation of a small examination volume (reduced FoV EPI sequence, e. g., ZOOMit by Siemens, iZOOM by Philips, or FOCUS by $G E$ ) or multi-shot sequences with segmented readout and susceptibility artifact/motion correction (readout segmented multishot EPI sequences, e. g., RESOLVE by Siemens or MUSE by GE) [34-36]. However, these new sequences need to be optimized for each MRI examination device to actually achieve an increase in quality compared to the usual SS-EPI sequence.

Healthy prostate tissue shows a high signal in DWI at low b-values and a significant signal decrease at high b-values. In contrast, prostate cancers with low water content and restricted particle mobility show low signal at low b-value and high signal at high b-value (diffusion restriction) [37]. The ADC map shows the signal difference semiquantitatively. In cases of prostate cancer, the ADC value is correspondingly reduced. As the biological aggressiveness of prostate cancers increases, their ADC value decreases [38]. However, the exact histopathological Gleason score for prostate cancer cannot be predicted. A decreasing ADC value within a prostate cancer in follow-up examinations with constant measurement parameters can be interpreted as an indication of increasing aggressiveness (e.g., in the context of active monitor- 
- Table 2 Advantages and disadvantages of axial angulation.

\begin{tabular}{|c|c|c|}
\hline Angulation & Advantages & Disadvantages \\
\hline $\begin{array}{l}1 \\
\text { Axial to urethra } \\
\text { The axial plane is angulated perpendicular } \\
\text { to the proximal intraprostatic course of } \\
\text { the urethra }\end{array}$ & $\begin{array}{l}\text { - Good correlation with the prostatectomy } \\
\text { specimen } \\
\text { - Anterior apical portions without partial volume } \\
\text { effects } \\
\text { - Symmetrical depiction of the transition zone }\end{array}$ & $\begin{array}{l}\text { - Dorsal apical partial volume effect } \\
\text { - Guidance of MTRA necessary, as sequence } \\
\text { planning is more difficult in cases of marked } \\
\text { hyperplasia or post-therapeutic changes } \\
\text { - Sections of several anatomical levels in one image }\end{array}$ \\
\hline $\begin{array}{l}2 \\
\text { Axial to longitudinal axis of the body } \\
\text { The axial plane is angulated perpendicular } \\
\text { to the longitudinal axis of the body }\end{array}$ & $\begin{array}{l}\text { - Simple and practicable } \\
\text { - High reproducibility in routine with continuous } \\
\text { examination without endorectal coil }\end{array}$ & $\begin{array}{l}\text { - Sections of several anatomical levels in one } \\
\text { image, making thus height assignment more } \\
\text { difficult (recommendation of sagittal plane as } \\
2^{\text {nd }} \text { plane) }\end{array}$ \\
\hline $\begin{array}{l}3 \\
\text { Axial to anterior rectal wall } \\
\text { The axial plane is angulated perpendicular } \\
\text { to the largest interface of the boundary } \\
\text { area and the anterior rectal wall. }\end{array}$ & $\begin{array}{l}\text { - Good correlation with TRUS images and with the } \\
\text { PI-RADS v2.1 sector scheme } \\
\text { - Peripheral zone vertical and dorsal border for the } \\
\text { most part depicted without partial volume effects }\end{array}$ & $\begin{array}{l}\text { - Partial volume effects at the boundary area of } \\
\text { the anterior portions of the peripheral zone } \\
\text { - Guidance of the MTRA necessary, because in } \\
\text { case of pronounced hyperplasia or post-thera- } \\
\text { peutic changes, sequence planning is more } \\
\text { difficult (prostate strongly convex shaped } \\
\text { dorsally and peripheral zone more difficult to } \\
\text { recognize due to compression) }\end{array}$ \\
\hline
\end{tabular}

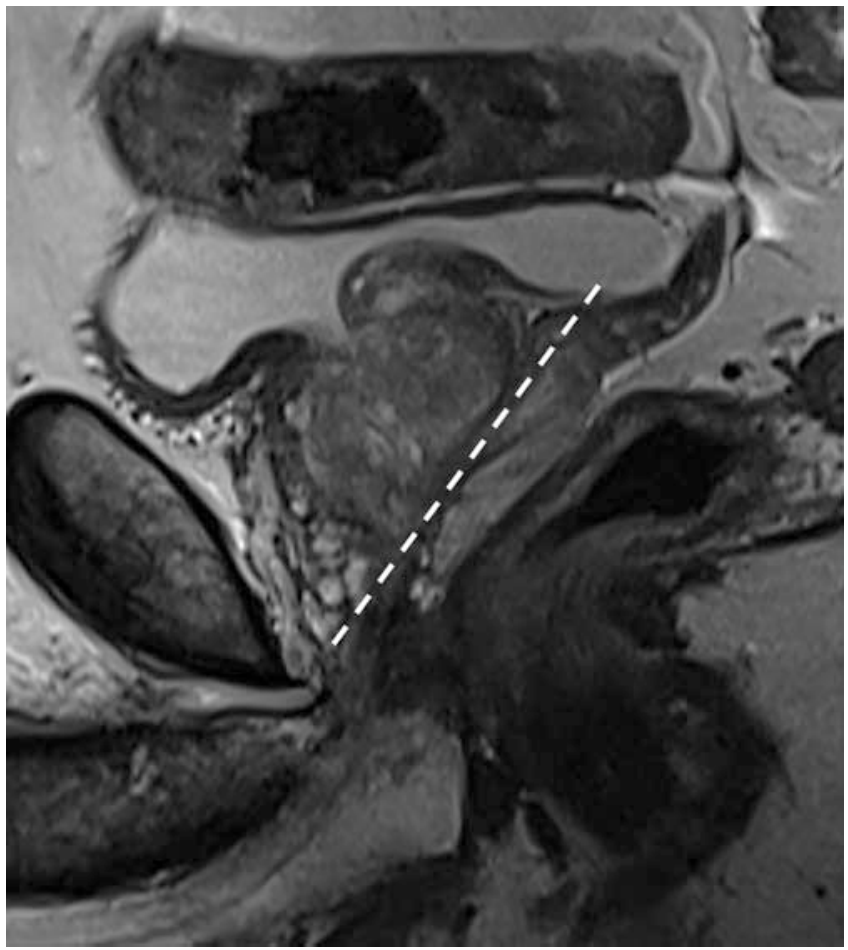

Fig. 2 Sagittal T2-weighted image for illustration of the coronar angulation.

ing). With external radiation therapy of the prostate, the ADC value increases significantly in prostate cancer, but does not change significantly in healthy prostate tissue [39].

The sensitivity and specificity of the DWI sequence alone for the detection of prostate cancer is reported to be 62 and $90 \%$, respectively, in a meta-analysis involving a total of 1204 patients [40]. In a meta-analysis with a total of 698 patients with previous negative prostate biopsy, the mean sensitivity of the DWI sequence for the detection of prostate cancer is $38 \%$ and the mean specificity is $95 \%$ [41]. The test quality parameters can be improved by combining the DWI with T2w and DCE sequences $[42,43]$.

\subsection{DCE sequence}

The DCE sequence involves repeated acquisition of fast T1w sequences during and after bolus intravenous application of a Gd-based contrast agent. High temporal resolution of $\leq 9$ seconds is a prerequisite for reliable imaging of early enhancement and maximal enhancement in prostate cancer and is preferable to high spatial resolution [44]. A measurement duration of preferably 3 min (but at least 2 min) in combination with the already described temporal resolution is necessary to sufficiently assess all characteristics of the signal intensity-time curve. A measurement time of $\geq 3 \mathrm{~min}$ is recommended for a stable calculation of pharmacokinetic parameter maps [45].

The images of the DCE sequence should initially be considered independently from those of the DWI and T2w sequences. Only in a second step should the abnormalities in the DCE sequence (e. g. early enhancement) be correlated with the images of the DWI and T2w sequences. In this context it should be noted that the importance of the DCE sequence is not limited to the upgrading of PI-RADS 3 lesions in the peripheral zone (upscoring), which alone can detect up to $33 \%$ clinically significant prostate cancers [46]. With respect to staging, the DCE sequence often allows a more precise representation of the volume of the index prostate cancer in comparison to the T2w or DWI sequence [47] and increases the diagnostic reliability for an infiltration of the seminal vesicles and the urinary bladder. A DCE sequence is essential for the diagnosis of recurrence after prostatectomy or after radiation therapy [48]. On the other hand, there are constellations in which the omission of DCE sequence does not lead to any diagnostic disadvantage for the patient. This is the case, for example, if, in the case of a clini- 
cally existing suspicion of carcinoma, the T2w and DWI sequences can be used to clearly identify the tumor and rule out focal therapy. In this context, reference is made to the notification from the German Federal Institute for Drugs and Medical Devices (BfArM) on gadolinium-based contrast agents dated January 11, 2018. Accordingly, doctors are advised to only use Gd-based contrast agents if essential diagnostic information cannot be obtained with magnetic resonance imaging without contrast enhancement [14]. Furthermore, a DCE sequence can be dispensed with if there are contraindications or the patient refuses the administration of a macrocyclic Gd-based contrast agent.

A bi-parametric prostate MRI (bpMRI) without the use of a DCE sequence cannot currently be generally recommended. The main reason is the insufficient evidence for bpMRI, as statements on the diagnostic value of MRI of the prostate rely mainly on data from mpMRI. Individual meta-analyses (the most recent is mentioned as an example), with a tendency towards a lower sensitivity of bPMRI ( $82 \%$ vs. $89 \%, p=0.39$ ), found no statistically significant differences for the sensitivity and specificity for the detection of prostate cancer between bPMRI and mPMRI of the prostate [49]. However, these statistically insignificant differences are based on the results of retrospective studies with heterogeneous protocols and radiologist experience and are limited in study design and their level of evidence [49]. Prospective studies justifying omission of the DCE sequence or accurately quantifying the differences have been lacking. Particularly in the case of less experienced examiners or possibly device-related poorer DWI sequence quality, the omission of a DCE sequence carries the risk that clinically significant prostate cancer will not be detected. It is undisputed that the DCE sequence increases diagnostic reliability and serves as a back-up sequence if the quality of the T2w or DWI sequence is unsuitable for diagnosis [20].

\subsection{T1w sequence}

A native T1w sequence of the entire pelvis can be a TSE/FSE or GRE sequence and should image the area from the aortic bifurcation to the pelvic floor either axially or coronally with a slice thickness of $\leq 5 \mathrm{~mm}$ (2D) or of $\leq 2 \mathrm{~mm}$ (3D). A DCE sequence also usually contains at least one native T1-weighted image before contrast administration, which can also be used diagnostically. In addition to the native T1w sequence, a T1w sequence can be carried out after contrast administration with a suitable fat saturation pulse.

$\mathrm{T} 1 \mathrm{w}$ images of the entire pelvis essentially have four tasks to fulfill. (1) Assessment of the T1w signal of the prostate and seminal vesicles, which indicate bleeding, protein-containing fluid in cysts, abscesses and calcifications; (2) Assessment of bone marrow signal to identify osteoblastic bone changes indicative of prostate cancer metastasis; (3) Detection, exact anatomical localization and (very limited) assessment of the status of enlarged lymph nodes; (4) Detection of other morphological changes in the pelvic organs and bones. Further information on the tasks of T1w images can be found in the "Supplementary Material" section.

\subsection{Endorectal coils}

A variety of factors affect imaging on the equipment side, including the main magnetic field strength, gradient amplitude, gradient slew rate, number of radio frequency transmitters, and coil technology. In addition to the usual rigid or flexible, flat-shaped external surface coils, there are also surface coils that can be worn experimentally, for example, the elements of which are positioned even closer to the prostate [50]. Alternatively, a combination of external surface coil and endorectal coil (ERC) can be used, whereby the SNR is increased for each magnetic field strength, which can be used for improved spatial resolution and/ or increased sequence speed. In addition to economic considerations, shorter sequence measurement times generally enable the acquisition of images with fewer movement artifacts with a higher concurrent resolution. The quality of the T2-weighted images, the DWI sequence with the diagnostically important high b values and the DCE sequence with high temporal resolution can be improved especially in patients with a high body mass index (BMI). Known disadvantages of previously predominantly used and single-use ERCs, such as increased cost, increased time, increased deformation of the prostate, increased artifacts, and local magnetic field inhomogeneity due to air in the balloon of the ERC, are predominantly offset by the development of new rigid reusable ERCs. In particular, the presacral position of a rigid ERC attached to a tripod-like construction without contact with the prostate and its smaller diameter are advantageous compared to the air-filled ERCs used up to now. Only the increased time and patient discomfort remain disadvantages when using a rigid ERC.

In general, the recommendation to use an ERC depends not only on the field strength, but also on other technical parameters of the MRI system used. The more powerful gradient systems used in newer MRI machines allow for a shorter echo time (TE), resulting in higher SNR and thus better image quality. Accordingly, the image quality of a 3 Tesla MRI unit with weak gradient system without combined ERC may be lower than the image quality of a 1.5 Tesla MRI unit with strong gradient system without combined ERC [21]. For the detection of clinically significant prostate cancer, the use of the combined endorectal surface coil system is still sometimes necessary on older 1.5 Tesla MRI machines and generally dispensable on modern 3 Tesla MRI machines. The literature available on this from the last decade is partly contradictory due to the large number of other non-comparable technical parameters [21, 50-53].

The use of an ERC with $3 \mathrm{~T}$ may be useful for special isues, such as local staging or assessment of extraprostatic extension, infiltration of the seminal vesicles, or the neurovascular bundle, but also for more precise localization of prostate cancer for indication and planning of focal therapy or for planning of focal boost irradiation during definitive radiotherapy.

\subsection{Additional sequences}

\section{${ }^{1} \mathrm{H}-\mathrm{MR}$ spectroscopy ( $\left.{ }^{1} \mathrm{H}-\mathrm{MRS}\right)$}

This established method is very well validated for the prostate, but it is associated with a high level of technical effort and is prone to artifacts. In combination with quality deficiencies in the auto- 
mation of post-processing and documentation steps, the spread of ${ }^{1} \mathrm{H}$-MRS in routine radiology is therefore limited. When used by experts, the three-dimensional ${ }^{1} \mathrm{H}$-MRS can reliably detect and localize prostate cancer [54]. A result of T2w imaging and ${ }^{1} \mathrm{H}$-MRS that are consistently suspicious for prostate cancer indicates the presence of prostate cancer with a probability of approx. $50 \%$ (positive predictive value) with the essential differential diagnosis of focal prostatitis. Conversely, a consistently negative result of T2w imaging and ${ }^{1} \mathrm{H}$-MRS indicates the presence of healthy prostate tissue with a probability of approx. 95\% (negative predictive value) with the differential diagnosis of diffuse prostatitis [55]. In a meta-analysis of 14 studies with a total of 698 patients with a previous negative prostate biopsy, the sensitivity and specificity of ${ }^{1} \mathrm{H}$-MRS combined with further MRI sequences for the detection of prostate cancer were $58 \%$ and $93 \%$, respectively [41]. The distinction between healthy and cancerous prostate tissue is basically retained in ${ }^{1} \mathrm{H}$-MRS even after therapy of the prostate (e. g. hormone therapy, radiotherapy, cryotherapy) [54].

\section{T2w 3 D multiecho sequences}

The contrast properties of the T2w $3 \mathrm{D}$ multiecho sequences are significantly different compared to classical T2w 2D TSE sequences. In addition, in-plane resolution analogous to $2 \mathrm{D}$ sequences can be achieved only with longer measuring time. However, individual studies on the detection of prostate cancer could not demonstrate any inferiority of the $3 \mathrm{D}$ sequence compared to the classic 2D sequences for the PI-RADS assessment [56]. Studies on local staging could even show a higher diagnostic accuracy of the $3 \mathrm{D}$ sequence with regard to expansion beyond the prostate [57]. However, this type of sequence should only be used in addition to standard T2w sequences. Because of the isotropic voxels, the 3D sequence may be advantageous for fusion with other imaging modalities (especially ultrasound, e.g., in the context of fusion biopsies with MRI-targeted, ultrasound-guided biopsy of the prostate).

\section{Advanced diffusion-weighted imaging techniques, such as diffusion tensor imaging (DTI) and diffusion kurtosis imaging (DKI)}

These techniques take into account the microstructural complexity of prostate cancer [58]. Intravoxel incoherent motion imaging takes into account the (non-linear) multi-exponential behavior of the diffusion signal at low b-values and thus the influence of the perfusion component on the signal. Diffusion kurtosis imaging takes into account the kurtosis of the tissue, which denotes the deviation of the diffusion signal from the Gaussian normal distribution (non-linearity of the diffusion signal at very high b values well above $1000 \mathrm{~s} / \mathrm{mm}^{2}$ ) [58, 59]. These complex diffusion models are currently the subject of research. The data currently available do not show any significant advantage of these methods over classic diffusion-weighted imaging, so these techniques are not part of routine diagnostics.
BOLD (blood oxygenation level-dependent) imaging, MR elastography, T1 and T2 mapping, arterial spin labeling (ASL)

These techniques are currently under development and are not part of routine diagnostics. T2 mapping as a method of quantitative assessment is promising in this regard, as prostate cancer has a shorter $\mathrm{T} 2$ relaxation time than the peripheral zone, although the diagnostic information gain appears to be lower for the transition zone compared with the peripheral zone [60].

\section{MRI in-bore biopsy}

\subsection{Indication and technique}

MRI/ultrasound fusion biopsy is the standard procedure for clarifying the abnormal areas described in the MRI. The targeted biopsy in the MRI device (MRI in-bore biopsy) is the only method that enables exact documentation of the removal location in the abnormal area. Because potential MRI/ultrasound fusion biopsy errors in segmentation (contouring of the prostate) and registration (localization of lesions) are eliminated, MRI in-bore biopsy is the most accurate MR-guided biopsy procedure for histological confirmation of MRI-diagnosed lesions [61-63]. The DynaTRIM (Philips Healthcare, Invivo Corporation) is the first approved MRI biopsy device. In addition, robotic-assisted MRI biopsy equipment from Soteria Medical (Remote Controlled Manipulator; RCM) is available. In patients with small or inconveniently located lesions, anal stenosis, coagulation disorders, or rectal disease (e. g., IBD), MRI in-bore biopsy may be preferred to MRI/ultrasound fusion biopsy after appropriate informed consent. After negative MRI/ultrasound fusion biopsy or after cognitive biopsy but (persistent) abnormal MRI findings (PI-RADS 4 and 5), targeted MRI in-bore biopsy within 6 months is useful (back-up procedure).

Possible access routes described in the literature are transrectal, transperineal and transgluteal. MRI in-bore biopsy should be performed under local anesthesia.

MRI-compatible biopsy needles (fully automatic or semi-automatic) should be used. The location of the biopsy needle and sampling site should be verified using a fast T2w sequence (e. g., single-shot TSE sequence) in two spatial planes (needle-in-scans).

Although there is no explicit approval of individual MRI-compatible biopsy needles at 1.5 Tesla and/or 3 Tesla, complication-free sampling has been demonstrated in studies at 3 Tesla using fully automated biopsy needles (150 mm and $175 \mathrm{~mm}$; Invivo) [63]. However, heating is possible depending on the needle length and field strength, which is why this should be explained accordingly to the patient.

Studies show high detection rates for patients without prior biopsy as well as with negative prior biopsy [64]. Sole MRI in-bore biopsy can be performed as an alternative to an MRI/US fusion biopsy in the secondary indication (after a negative biopsy) [65]. MRI in-bore and fusion-based MRI/ultrasound biopsy procedures tend to be superior to simple cognitive biopsy, but there are no valid prospective comparative data [66, 67]. 


\subsection{Laboratory procedures}

Current laboratory parameters should be determined according to the current recommendations of the Cardiovascular and Interventional Radiological Society of Europe (CIRSE) [68]. However, conventional coagulation diagnostics (international normalized ratio (INR); activated partial thromboplastin time (aPTT); platelet count) are limited in detecting blood coagulation disorders such as Von Willebrand factor deficiency or platelet dysfunction [69]. A bleeding history as well as recording of previous surgery, trauma, familial bleeding tendency and anticoagulant medication should be performed. Urinalysis can be carried out in advance to rule out a urinary tract infection. A biopsy should be avoided if there are clinical signs of an acute urinary tract infection (e. g. burning sensation when urinating). A rectal swab can be taken beforehand to determine resistance to antibiotics.

\subsection{Anticoagulant medication}

Prostate biopsy belongs to risk group 1 with a low risk of bleeding (Consensus Guidelines of CIRSE) [68] even though, strictly speaking, it is not usually performed percutaneously. The INR value should be $\leq 2.0$ and the platelet count $\geq 50000$.

The use of anticoagulants is usually not a contraindication for risk group 1 [68]. Taking acetylsalicylic acid (ASA) can potentially increase the risk of bleeding complications, but a dose of $100 \mathrm{mg}$ p. o. in particular does not have to be terminated beforehand [69, 70]. Dual anti-platelet therapy (ASA + ADP antagonists [clopidogrel]), on the other hand, should be converted to monotherapy with ASA in interdisciplinary consultation [71]. Vitamin K antagonists (Marcumar) should be discontinued 3-5 days in advance, if possible [68]. New anticoagulants such as dabigatran, rivaroxaban, or apixaban should be discontinued at least 24 hours beforehand [72].

\subsection{Antibiotics and anesthesia}

Based on the recommendations of the $\mathrm{S3}$ guideline for prostate cancer on transrectal ultrasound-guided biopsy, it is recommended that the transrectal MRI in-bore biopsy be performed under antibiotic protection. Antibiotic prophylaxis was shown to significantly decrease the rate of bacteriuria after punch biopsy as a possible surrogate parameter for infection [73]. In addition, the typically smaller number of biopsy cylinders used in MRI biopsy appears to contribute to a lower infection rate. An increase in infectious complication after prostate biopsy has been described in recent years [74]. On April 30, 2019, the German Medicines Agency (BfArM) revoked indications for systemic use (e.g. for the prophylaxis of urinary tract infections during transrectal prostate biopsy) of all fluoroquinolones approved in the EU (active ingredients concerned: ciprofloxacin, levofloxacin, moxifloxacin, norfloxacin, ofloxacin) due to the risk of relevant side effects (muscles, joints and nervous system). Alternatives are cephalosporins (e.g., cefpodoxime, cefixime), fosfomycin, or aminoglycosides (e. g., gentamicins). Single-shot intravenous antibiotics of a cephalosporin (ceftriaxone; Cefotrix [D, A], Rocephin [D, A, CH], Tercefon $[A]$ ) can be given one hour before intervention.
When performed routinely, the patient has good to very good pain analgesia by means of gel anesthesia during the transrectal MRI biopsy [63].

\section{Conflict of Interest}

TF: Research funding: Central Innovation Program for SMEs of the Federal Ministry for Economic Affairs and Energy (ZF4816001 BA9); Lecture fees: Saegeling Medizintechnik GmbH, Bayer AG, Medac Gmbh; Expert Council: Bayer AG DB: Patent holder MRT-in-bore biopsy. SK: Council of Experts: Bayer AG other authors: no conflicts.

\section{Literatur}

[1] Leitlinienprogramm Onkologie. Interdisziplinäre Leitlinie der Qualität S3 zur Früherkennung, Diagnose und Therapie der verschiedenen Stadien des Prostatakarzinoms Version 5.1. 2019

[2] Distler FA, Radtke JP, Bonekamp D et al. The Value of PSA Density in Combination with PI-RADS for the Accuracy of Prostate Cancer Prediction. J Urol 2017; 198: 575-582. doi:10.1016/j.juro.2017.03.130

[3] Gupta RT, Brown AF, Silverman RK et al. Can Radiologic Staging With Multiparametric MRI Enhance the Accuracy of the Partin Tables in Predicting Organ-Confined Prostate Cancer? Am J Roentgenol 2016; 207: 87-95. doi:10.2214/AJR.15.15878

[4] Lai WS, Gordetsky JB, Thomas JV et al. Factors predicting prostate cancer upgrading on magnetic resonance imaging-targeted biopsy in an active surveillance population. Cancer 2017; 123: 1941-1948. doi:10.1002/ cncr.30548

[5] Radtke JP, Wiesenfarth M, Kesch C et al. Combined Clinical Parameters and Multiparametric Magnetic Resonance Imaging for Advanced Risk Modeling of Prostate Cancer-Patient-tailored Risk Stratification Can Reduce Unnecessary Biopsies. Eur Urol 2017; 72: 888-896. doi:10.1016/ j.eururo.2017.03.039

[6] Stonier T, Simson N, Shah T et al. The "Is mpMRI Enough" or IMRIE Study: A Multicentre Evaluation of Prebiopsy Multiparametric Magnetic Resonance Imaging Compared with Biopsy. Eur Urol Focus 2020. doi:10.1016/j.euf.2020.09.012

[7] Robert Koch Institut (RKI) und Gesellschaft der epidemiologischen Krebsregister in Deutschland (GEKID). Krebs in Deutschland für 2013/14. Berlin, 2017

[8] Zeegers MP, Jellema A, Ostrer H. Empiric risk of prostate carcinoma for relatives of patients with prostate carcinoma: a meta - analysis. Cancer 2003; 97: 1894-1903

[9] Mueller-Lisse UG, Swanson MG, Vigneron DB et al. Time-dependent effects of hormone-deprivation therapy on prostate metabolism as detected by combined magnetic resonance imaging and 3D magnetic resonance spectroscopic imaging. Magn Reson Med 2001; 46: 49-57. doi: $10.1002 / \mathrm{mrm} .1159$

[10] Truong H, Logan J, Turkbey B et al. MRI characterization of the dynamic effects of 5alpha-reductase inhibitors on prostate zonal volumes. Can J Urol 2013; 20: 7002-7007

[11] Franiel T, Ludemann L, Taupitz M et al. MRI before and after external beam intensity-modulated radiotherapy of patients with prostate cancer: the feasibility of monitoring of radiation-induced tissue changes using a dynamic contrast-enhanced inversion-prepared dual-contrast gradient echo sequence. Radiother Oncol 2009; 93: 241-245. doi:10.1016/j.radonc.2009.08.016

[12] Franiel T, Ludemann L, Rudolph B et al. Evaluation of normal prostate tissue, chronic prostatitis, and prostate cancer by quantitative perfusion analysis using a dynamic contrast-enhanced inversion-prepared dual-contrast gradient echo sequence. Invest Radiol 2008; 43: 481-487. doi:10.1097/RLI.0b013e31816b2f63 
[13] European Society of Urogenital Radiology. ESUR Guidelines on Contrast Agents 10.0. 2018

[14] Europäische Arzneimittelagentur (EMA) und Bundesinstitut für Arzneimittel und Medizinprodukte (BfArM). Gadolinium-haltige Kontrastmittel: Aktualisierte Empfehlungen nach Bewertung von Gadoliniumablagerungen im Gehirn und anderen Geweben 2018.

[15] Tsai LL, Grant AK, Mortele KJ et al. A Practical Guide to MR Imaging Safety: What Radiologists Need to Know. Radiographics 2015; 35 : 1722-1737. doi:10.1148/rg.2015150108

[16] Attenberger UI, Rathmann N, Sertdemir M et al. Small Field-of-view single-shot EPI-DWI of the prostate: Evaluation of spatially-tailored two-dimensional radiofrequency excitation pulses. Z Med Phys 2016; 26: 168-176. doi:10.1016/j.zemedi.2015.06.013

[17] Czarniecki M, Caglic I, Grist JT et al. Role of PROPELLER-DWI of the prostate in reducing distortion and artefact from total hip replacement metalwork. Eur J Radiol 2018; 102: 213-219. doi:10.1016/j.ejrad.2018.03.021

[18] Ariyanayagam T, Malcolm PN, Toms AP. Advances in Metal Artifact Reduction Techniques for Periprosthetic Soft Tissue Imaging. Semin Musculoskel R 2015; 19: 328-334. doi:10.1055/s-0035-1563734

[19] Barrett T, Vargas H, Akin O et al. Value of the hemorrhage exclusion sign on T1-weighted prostate MR images for the detection of prostate cancer. Radiology 2012; 263: 751-757

[20] European Society of Urogenital Radioloy, American College of Radiology. Prostate Imaging - Reporting and Data System Version 2.1. 2019

[21] Engels RRM, Israel B, Padhani AR et al. Multiparametric Magnetic Resonance Imaging for the Detection of Clinically Significant Prostate Cancer: What Urologists Need to Know. Part 1: Acquisition. Eur Urol 2020; 77: 457-468. doi:10.1016/j.eururo.2019.09.021

[22] Slough RA, Caglic I, Hansen NL et al. Effect of hyoscine butylbromide on prostate multiparametric MRI anatomical and functional image quality. Clin Radiol 2018; 73: 216 e219-216 e214. doi:10.1016/j. crad.2017.07.013

[23] Ullrich T, Quentin M, Schmaltz AK et al. Hyoscine butylbromide significantly decreases motion artefacts and allows better delineation of anatomic structures in mp-MRI of the prostate. Eur Radiol 2018; 28: 17-23. doi:10.1007/s00330-017-4940-7

[24] Roethke MC, Kuru TH, Radbruch A et al. Prostate magnetic resonance imaging at 3 Tesla: Is administration of hyoscine-N-butyl-bromide mandatory? World J Radiol 2013; 5: 259-263. doi:10.4329/wjr.v5.i7.259

[25] Wagner M, Rief M, Busch J et al. Effect of butylscopolamine on image quality in MRI of the prostate. Clin Radiol 2010; 65: 460-464. doi:10.1016/j.crad.2010.02.007

[26] Caglic I, Hansen NL, Slough RA et al. Evaluating the effect of rectal distension on prostate multiparametric MRI image quality. Eur J Radiol 2017; 90: 174-180. doi:10.1016/j.ejrad.2017.02.029

[27] Lim C, Quon J, McInnes M et al. Does a cleansing enema improve image quality of 3T surface coil multiparametric prostate MRI? J Magn Reson Imaging 2015; 42: 689-697. doi:10.1002/jmri.24833

[28] Medved M, Sammet S, Yousuf A et al. MR imaging of the prostate and adjacent anatomic structures before, during, and after ejaculation: qualitative and quantitative evaluation. Radiology 2014; 271: 452-460. doi:10.1148/radiol.14131374

[29] Kabakus IM, Borofsky S, Mertan FV et al. Does Abstinence From Ejaculation Before Prostate MRI Improve Evaluation of the Seminal Vesicles? Am J Roentgenol 2016; 207: 1205-1209. doi:10.2214/AJR.16.16278

[30] Barrett T, Tanner ], Gill AB et al. The longitudinal effect of ejaculation on seminal vesicle fluid volume and whole-prostate $A D C$ as measured on prostate MRI. Eur Radiol 2017; 27: 5236-5243. doi:10.1007/s00330017-4905-x

[31] Shin T, Kaji Y, Shukuya T et al. Significant changes of T2 value in the peripheral zone and seminal vesicles after ejaculation. Eur Radiol 2018; 28: 1009-1015. doi:10.1007/s00330-017-5077-4
[32] Yuruk E, Pastuszak AW, Suggs JM 3rd et al. The association between seminal vesicle size and duration of abstinence from ejaculation. Andrologia 2017; 49: doi:10.1111/and.12707

[33] Mueller-Lisse UG, Mueller-Lisse UL, Zamecnik P et al. [Diffusion-weighted MRI of the prostate]. Radiologe 2011; 51: 205-214. doi:10.1007/s00117010-2061-2

[34] Klingebiel M, Ullrich T, Quentin M et al. Advanced diffusion weighted imaging of the prostate: Comparison of readout-segmented multi-shot, parallel-transmit and single-shot echo-planar imaging. Eur J Radiol 2020; 130: 109161 doi:10.1016/j.ejrad.2020.109161

[35] Stocker D, Manoliu A, Becker AS et al. Image Quality and Geometric Distortion of Modern Diffusion-Weighted Imaging Sequences in Magnetic Resonance Imaging of the Prostate. Invest Radiol 2018; 53: 200206. doi:10.1097/rli.0000000000000429

[36] Thierfelder KM, Scherr MK, Notohamiprodjo M et al. Diffusion-weighted MRI of the prostate: advantages of Zoomed EPI with parallel-transmitaccelerated 2D-selective excitation imaging. Eur Radiol 2014; 24: $3233-$ 3241. doi:10.1007/s00330-014-3347-y

[37] Quentin M, Schimmoller L, Arsov C et al. Increased signal intensity of prostate lesions on high b-value diffusion-weighted images as a predictive sign of malignancy. Eur Radiol 2014; 24: 209-213. doi:10.1007/ s00330-013-2999-3

[38] Vargas HA, Akin O, Franiel T et al. Diffusion-weighted endorectal MR imaging at $3 \mathrm{~T}$ for prostate cancer: tumor detection and assessment of aggressiveness. Radiology 2011; 259: 775-784. doi:10.1148/radiol. 11102066

[39] Decker G, Murtz P, Gieseke J et al. Intensity-modulated radiotherapy of the prostate: dynamic ADC monitoring by DWI at 3.0 T. Radiother Oncol 2014; 113: 115-120. doi:10.1016/j.radonc.2014.07.016

[40] Jie C, Rongbo L, Ping T. The value of diffusion-weighted imaging in the detection of prostate cancer: a meta-analysis. Eur Radiol 2014; 24 : 1929-1941. doi:10.1007/s00330-014-3201-2

[41] Zhang ZX, Yang J, Zhang CZ et al. The value of magnetic resonance imaging in the detection of prostate cancer in patients with previous negative biopsies and elevated prostate-specific antigen levels: a meta-analysis. Acad Radiol 2014; 21: 578-589. doi:10.1016/ j.acra.2014.01.004

[42] Schimmoller L, Quentin M, Arsov C et al. MR-sequences for prostate cancer diagnostics: validation based on the PI-RADS scoring system and targeted MR-guided in-bore biopsy. Eur Radiol 2014; 24: 2582-2589. doi:10.1007/s00330-014-3276-9

[43] Wu LM, Xu JR, Ye YQ et al. The clinical value of diffusion-weighted imaging in combination with $\mathrm{T} 2$-weighted imaging in diagnosing prostate carcinoma: a systematic review and meta-analysis. Am J Roentgenol 2012; 199: 103-110. doi:10.2214/ajr.11.7634

[44] Othman AE, Falkner F, Weiss J et al. Effect of Temporal Resolution on Diagnostic Performance of Dynamic Contrast-Enhanced Magnetic Resonance Imaging of the Prostate. Invest Radiol 2016; 51: 290-296. doi:10.1097/rli.0000000000000234

[45] Othman AE, Falkner F, Martirosian P et al. Optimized Fast Dynamic Contrast-Enhanced Magnetic Resonance Imaging of the Prostate: Effect of Sampling Duration on Pharmacokinetic Parameters. Invest Radiol 2016; 51: 106-112. doi:10.1097/rli.0000000000000213

[46] Ullrich T, Quentin M, Arsov C et al. Value of Dynamic Contrast-Enhanced (DCE) MR Imaging in Peripheral Lesions in PI-RADS-4 Patients. Rofo 2020; 192: 441-447. doi:10.1055/a-1020-4026

[47] Sun C, Chatterjee A, Yousuf A et al. Comparison of T2-Weighted Imaging, DWI, and Dynamic Contrast-Enhanced MRI for Calculation of Prostate Cancer Index Lesion Volume: Correlation With Whole-Mount Pathology. Am J Roentgenol 2019; 212: 351-356. doi:10.2214/ ajr.18.20147

[48] Barret E, Turkbey B, Puech P et al. Update on the ICUD-SIU consultation on multi-parametric magnetic resonance imaging in localised prostate 
cancer. World J Urol 2019; 37: 429-436. doi:10.1007/s00345-0182395-3

[49] Bass E], Pantovic A, Connor $M$ et al. A systematic review and meta-analysis of the diagnostic accuracy of biparametric prostate MRI for prostate cancer in men at risk. Prostate Cancer and Prostatic Diseases 2020. doi:10.1038/s41391-020-00298-w; online ahead of prin

[50] O'Donohoe RL, Dunne RM, Kimbrell V et al. Prostate MRI using an external phased array wearable pelvic coil at 3T: comparison with an endorectal coil. Abdom Radiol (NY) 2019; 44: 1062-1069. doi:10.1007| s00261-018-1804-9

[51] Baur AD, Daqqaq T, Wagner $M$ et al. T2- and diffusion-weighted magnetic resonance imaging at $3 \mathrm{~T}$ for the detection of prostate cancer with and without endorectal coil: An intraindividual comparison of image quality and diagnostic performance. Eur J Radiol 2016; 85: 1075-1084. doi:10.1016/j.ejrad.2016.03.014

[52] Shah ZK, Elias SN, Abaza R et al. Performance comparison of 1.5-T endorectal coil MRI with 3.0-T nonendorectal coil MRI in patients with prostate cancer. Acad Radiol 2015; 22: 467-474. doi:10.1016/j. acra.2014.11.007

[53] Turkbey B, Merino M], Gallardo EC et al. Comparison of endorectal coil and nonendorectal coil T2W and diffusion-weighted MRI at 3 Tesla for localizing prostate cancer: correlation with whole-mount histopathology. J Magn Reson Imaging 2014; 39: 1443-1448. doi:10.1002/jmri.24317

[54] Mueller-Lisse UG, Scherr MK. Proton MR spectroscopy of the prostate. Eur J Radiol 2007; 63: 351-360. doi:10.1016/j.ejrad.2007.06.024

[55] Umbehr M, Bachmann LM, Held U et al. Combined magnetic resonance imaging and magnetic resonance spectroscopy imaging in the diagnosis of prostate cancer: a systematic review and meta-analysis. Eur Urol 2009; 55: 575-590. doi:10.1016/j.eururo.2008.10.019

[56] Polanec SH, Lazar M, Wengert G] et al. 3D T2-weighted imaging to shorten multiparametric prostate MRI protocols. Eur Radiol 2018; 28 : 1634-1641. doi:10.1007/s00330-017-5120-5

[57] Caglic I, Povalej BrzanP, Warren AY et al. Defining the incremental value of 3D T2-weighted imaging in the assessment of prostate cancer extracapsular extension. Eur Radiol 2019; 29: 5488-5497. doi:10.1007| s00330-019-06070-6

[58] Ding K, Yao Y, Gao Y et al. Diagnostic evaluation of diffusion kurtosis imaging for prostate cancer: Detection in a biopsy population. Eur ] Radiol 2019; 118: 138-146. doi:10.1016/j.ejrad.2019.07.009

[59] Roethke MC, Kuder TA, Kuru TH et al. Evaluation of Diffusion Kurtosis Imaging Versus Standard Diffusion Imaging for Detection and Grading of Peripheral Zone Prostate Cancer. Invest Radiol 2015; 50: 483-489. doi:10.1097/rli.0000000000000155

[60] Mai ], Abubrig M, Lehmann T et al. T2 Mapping in Prostate Cancer. Investigative Radiology 2019; 54: 146-152

[61] Kaufmann S, Russo GI, Bamberg F et al. Prostate cancer detection in patients with prior negative biopsy undergoing cognitive-, robotic- or in-bore MRI target biopsy. World J Urol 2018; 36: 761-768. doi:10.1007| s00345-018-2189-7

[62] Oberlin DT, Casalino DD, Miller FH et al. Diagnostic Value of Guided Biopsies: Fusion and Cognitive-registration Magnetic Resonance Imaging Versus Conventional Ultrasound Biopsy of the Prostate. Urology 2016; 92: 75-79. doi:10.1016/j.urology.2016.02.041
[63] Schimmoller L, Blondin D, Arsov C et al. MRI-Guided In-Bore Biopsy: Differences Between Prostate Cancer Detection and Localization in Primary and Secondary Biopsy Settings. Am J Roentgenol 2016; 206: 92-99. doi:10.2214/ajr.15.14579

[64] Kaufmann S, Kruck S, Kramer U et al. Direct comparison of targeted MRI-guided biopsy with systematic transrectal ultrasound-guided biopsy in patients with previous negative prostate biopsies. Urol Int 2015; 94: 319-325. doi:10.1159/000365397

[65] Arsov C, Rabenalt R, Blondin D et al. Prospective randomized trial comparing magnetic resonance imaging (MRI)-guided in-bore biopsy to MRI-ultrasound fusion and transrectal ultrasound-guided prostate biopsy in patients with prior negative biopsies. Eur Urol 2015; 68: 713720. doi:10.1016/j.eururo.2015.06.008

[66] Durmus T, Reichelt U, Huppertz A et al. MRI-guided biopsy of the prostate: correlation between the cancer detection rate and the number of previous negative TRUS biopsies. Diagn Interv Radiol 2013; 19: 411417. doi:10.5152/dir.2013.13055

[67] Rastinehad AR, Durand M. A comparison of magnetic resonance imaging and ultrasonography (MRI/US)-fusion guided prostate biopsy devices: too many uncontrolled variables. BJU Int 2016; 117: 548-549. doi:10.1111/bju. 13344

[68] Patel IJ, Rahim S, Davidson JC et al. Society of Interventional Radiology Consensus Guidelines for the Periprocedural Management of Thrombotic and Bleeding Risk in Patients Undergoing Percutaneous ImageGuided Interventions-Part II: Recommendations: Endorsed by the Canadian Association for Interventional Radiology and the Cardiovascular and Interventional Radiological Society of Europe. J Vasc Interv Radiol 2019; 30: 1168-1184 e1161. doi:10.1016/j.jvir.2019.04.017

[69] Chee YL, Crawford JC, Watson HG et al. Guidelines on the assessment of bleeding risk prior to surgery or invasive procedures. British Committee for Standards in Haematology. Br J Haematol 2008; 140: 496-504. doi:10.1111/j.1365-2141.2007.06968.x

[70] Burger W, Chemnitius JM, KneissI GD et al. Low-dose aspirin for secondary cardiovascular prevention - cardiovascular risks after its perioperative withdrawal versus bleeding risks with its continuation - review and meta-analysis. J Intern Med 2005; 257: 399-414. doi:10.1111/j.13652796.2005.01477.x

[71] Poldermans D, Bax J], Boersma E et al. Guidelines for pre-operative cardiac risk assessment and perioperative cardiac management in noncardiac surgery: the Task Force for Preoperative Cardiac Risk Assessment and Perioperative Cardiac Management in Non-cardiac Surgery of the European Society of Cardiology (ESC) and endorsed by the European Society of Anaesthesiology (ESA). Eur J Anaesthesiol 2010; 27: 92-137. doi:10.1097/EJA.0b013e328334c017

[72] Patel IJ, Davidson JC, Nikolic B et al. Addendum of newer anticoagulants to the SIR consensus guideline. J Vasc Interv Radiol 2013; 24: 641-645. doi:10.1016/j.jvir.2012.12.007

[73] Bootsma AM, Laguna Pes MP, Geerlings SE et al. Antibiotic prophylaxis in urologic procedures: a systematic review. Eur Urol 2008; 54: $1270-$ 1286. doi:10.1016/j.eururo.2008.03.033

[74] Loeb S, Carter HB, Berndt SI et al. Complications after prostate biopsy: data from SEER-Medicare. J Urol 2011; 186: 1830-1834. doi:10.1016/ j.juro.2011.06.057 